\title{
Blow-up for the weakly dissipative generalized Camassa-Holm equation
}

\author{
Yantao Guo ${ }^{1,2}$ and Yanbin Tang ${ }^{1 *}$
}

"Correspondence:

tangybhust@sina.com

'school of Mathematics and

Statistics, Huazhong University of

Science and Technology, Wuhan, Hubei 430074, P.R. China

Full list of author information is

available at the end of the article

\begin{abstract}
The main goal of this paper is to investigate the blow-up phenomena of solutions to a weakly dissipative generalized Camassa-Holm equation, which contains higher power nonlinear dispersion terms and a convection term. We give a sufficient condition on the initial data such that the strong solution blows up at a finite time, and then we establish an estimate of the blow-up time. Finally, we give a global existence result of the strong solution.
\end{abstract}

MSC: 35A35; 35B30; 35G25; 35Q53

Keywords: generalized Camassa-Holm equation; weak dissipativity; blow-up; global existence

\section{Introduction}

In recent years, following the research of the Burgers equation, the $\mathrm{KdV}$ equation, and the BBM equation [1], the generalized Camassa-Holm equation

$$
u_{t}-u_{x x t}+(k+2) u^{k} u_{x}-(k+1) u^{k-1} u_{x} u_{x x}-u^{k} u_{x x x}=0
$$

has attracted much attention in the study of mathematical physics, where $k \geq 1, k \in N$.

For $k=1,(1.1)$ is reduced to the classical Camassa-Holm equation

$$
u_{t}-u_{x x t}+3 u u_{x}-2 u_{x} u_{x x}-u u_{x x x}=0,
$$

which describes the unidirectional propagation of waves at the free surface of shallow water. $u(t, x)$ stands for the fluid velocity at time $t$ in the spatial direction $x$. The CamassaHolm equation (1.2) is bi-Hamiltonian and admits an infinite number of conservation laws [2]. The Camassa-Holm equation (1.2) has been extensively studied by Constantin and Escher [3-6], Lai and Wu [7], and so on. The well-posedness of the Camassa-Holm shallow water equation has been established, and some blow-up scenarios were derived by Constantin and Escher [8], Wu and Yin [9, 10], Lai and Wu [11], Zhou [12], Xin and Zhang [13, 14].

For $k=2$, (1.1) becomes the Novikov equation

$$
u_{t}-u_{x x t}+4 u^{2} u_{x}-3 u u_{x} u_{x x}-u^{2} u_{x x x}=0
$$

○2014 Guo and Tang; licensee Springer. This is an Open Access article distributed under the terms of the Creative Commons Attribution License (http://creativecommons.org/licenses/by/2.0), which permits unrestricted use, distribution, and reproduction in any medium, provided the original work is properly cited. 
which was recently discovered by Novikov [15]. Since the Novikov equation possesses a matrix Lax pair and has a bi-Hamiltonian structure as the Camassa-Holm equation, this equation has been studied by many researchers in the past few years, the well-posedness and persistence properties were studied by Lai et al. [16], Ni and Zhou [17], Zhao et al. [18]. Jiang and $\mathrm{Ni}$ [19] considered the blow-up phenomena for the integrable Novikov equation, Yan et al. [20] gave the global existence and blow-up phenomena for the weakly dissipative Novikov equation.

In this paper, we investigate the Cauchy problem for a generalized weakly dissipative Camassa-Holm equation

$$
\left\{\begin{array}{l}
u_{t}-u_{x x t}+(k+2) u^{k} u_{x}-(k+1) u^{k-1} u_{x} u_{x x}-u^{k} u_{x x x}+\lambda\left(u-u_{x x}\right)=0 \\
u(0, x)=u_{0}(x), \quad x \in R
\end{array}\right.
$$

where $\lambda>0, k \geq 1$ is a positive integer. Equations (1.4) and (1.1) have similar properties as regards the local well-posedness and blow-up phenomena, but they are different as regards the long time behavior. For example, when $k=1,(1.1)$ is completely integrable and has an infinite number of conservation laws, but for the corresponding equation (1.4), $\int\left(u^{2}+u_{x}^{2}\right) d x$ is not conservative.

Zhao et al. [21] studied the existence of global weak solutions to the Cauchy problem of the generalized Novikov equation (1.1). Liu and Yin [22] investigated the blow-up phenomena for the Degasperis-Procesi equation

$$
u_{t}-u_{t x x}+4 u u_{x}=3 u_{x} u_{x x}+u u_{x x x}, \quad t>0, x \in R,
$$

it is very similar with (1.4), but (1.4) contains the higher power nonlinear dispersion terms $(k+1) u^{k-1} u_{x} u_{x x}, u^{k} u_{x x x}$, and the nonlinear convection term $(k+2) u^{k} u_{x}$.

Compared to [22], the main difficulty in this paper comes from the nonlinear effect of higher power nonlinear dispersion terms $(k+1) u^{k-1} u_{x} u_{x x}, u^{k} u_{x x x}$, and the nonlinear convection term $(k+2) u^{k} u_{x}$. On the other hand, in the proof of the blow-up property of the solution to (1.4), we need the sign of the term $u^{k-2}(t, x)$, but $u(t, x)$ changes the sign for $x \in R$. Compared to the classical Camassa-Holm equation $(k=1)$ and the classical Novikov equation $(k=2)$, the term $u^{k-2}(t, x)$ disappears, accordingly. Therefore, we generalized the blow-up property of solutions to the Cauchy problem (1.4).

We first give a sufficient condition on the initial data such that the strong solution of (1.4) blows up at a finite time, and then we establish an estimate of the blow-up time. Finally, we give a global existence result of the strong solution of (1.4).

The paper is organized as follows. In Section 2, we give some preliminaries used in our investigation. In Section 3, we give in our main conclusion the blow-up scenario and global existence result.

\section{Preliminaries}

We first review some notations. The convolution between two functions $f(x)$ and $g(x)$ :

$$
(f * g)(x)=\int_{R} f(x-y) g(y) d y, \quad \forall f, g \in \mathcal{S}
$$

where $\mathcal{S}$ is the Schwartz class. For any $f(x) \in \mathcal{S}$, the Fourier transform of $f(x)$ is defined by $\mathcal{F}(f(x))=\hat{f}(\xi)$, the inverse Fourier transform of $\hat{f}(\xi)$ denoted by $\mathcal{F}^{-1}(\hat{f}(\xi))$. If $f(x) \in H^{s}$, 
$s \in R$, then the norm of $f(x)$ is

$$
\|f\|_{H^{s}}=\left(\int_{R}\left(1+|\xi|^{2}\right)^{s}|\hat{f}(\xi)|^{2} d \xi\right)^{\frac{1}{2}}
$$

Set $y=u-u_{x x}$, the Cauchy problem (1.4) becomes

$$
\left\{\begin{array}{l}
y_{t}+u^{k} y_{x}+(k+1) u^{k-1} u_{x} y+\lambda y=0, \quad x \in R, t>0 \\
u(0, x)=u_{0}(x), \quad x \in R
\end{array}\right.
$$

Since $G(x)=\frac{1}{2} e^{-|x|}$ is the Green's function of the differential equation $u-u_{x x}=\delta(x)$, for all $f(x) \in L^{2}(R), G * f(x)=\left(1-\partial_{x}^{2}\right)^{-1} f(x)$, and $G * y=u(t, x)$, and thus the Cauchy problem (2.1) can be rewritten as

$$
\left\{\begin{array}{l}
u_{t}+u^{k} u_{x}+G *\left[k(k-1) u^{k-2} u_{x}^{3}+(2 k-1) u^{k-1} u_{x} u_{x x}+(k+1) u^{k} u_{x}\right]+\lambda u=0 \\
u(0, x)=u_{0}(x)
\end{array}\right.
$$

Zhao et al. [18, 21] gave the local and global existence of solutions to the Cauchy problem (2.2), it is crucial in our discussion.

Lemma 2.1 [18] Given $u_{0}(x) \in H^{s}(R), s>\frac{3}{2}$, then there exist a constant $T=T\left(u_{0}\right)>0$ and a unique solution $u(t, x)$ to $(2.2)$ such that

$$
u(t, x) \in C\left([0, T) ; H^{s}(R)\right) \cap C^{1}\left([0, T) ; H^{s-1}(R)\right) .
$$

Moreover, the mapping $u_{0} \rightarrow u\left(\cdot, u_{0}\right): H^{s}(R) \rightarrow C\left([0, T) ; H^{s}(R)\right) \cap C^{1}\left([0, T) ; H^{s-1}(R)\right)$ is Hölder continuous.

We now describe some properties of solutions of the following initial value problem:

$$
\left\{\begin{array}{l}
\frac{\partial q}{\partial t}(t, x)=u^{k}(t, q(t, x)), \quad t>0, x \in R \\
q(0, x)=x, \quad x \in R
\end{array}\right.
$$

where $u(t, x)$ is a solution to the Cauchy problem (2.2). The following important properties are immediate consequence of the classical results in the theory of ordinary differential equations.

Lemma 2.2 Let $u_{0}(x) \in H^{s}(R), s>\frac{3}{2}$, and $T=T\left(u_{0}\right)>0$ be the maximal existence time of the corresponding solution $u(t, x)$ to (2.2), then the problem (2.3) has a unique solution $q \in C^{1}([0, T) \times R ; R)$. Moreover, the map $q(t, \cdot)$ is an increasing diffeomorphism of $R$ with

$$
q_{x}(t, x)=\exp \left(k \int_{0}^{t} u^{k-1} u_{x}(s, x) d s\right), \quad(t, x) \in[0, T) \times R .
$$

Lemma 2.3 Let $u_{0}(x) \in H^{s}(R), s>\frac{3}{2}$, and $T=T\left(u_{0}\right)>0$ be the maximal existence time of the corresponding solution $u(t, x)$ to $(2.2)$. For $y(t, x)=u-u_{x x}$, we have

$$
y(t, q(t, x)) q_{x}^{\frac{k+1}{k}}(t, x)=y_{0}(x) e^{-\lambda t}, \quad(t, x) \in[0, T) \times R .
$$


Proof Let $P(t)=y(t, q(t, x)) q_{x}^{\frac{k+1}{k}}(t, x)$. Thanks to (2.1) and (2.3), we have

$$
\begin{aligned}
\frac{d P}{d t}= & y_{t}(t, q(t, x)) q_{x}^{\frac{k+1}{k}}(t, x)+y_{x}(t, q(t, x)) q_{t}(t, x) q_{x}^{\frac{k+1}{k}}(t, x) \\
& +\frac{k+1}{k} y(t, q(t, x)) q_{x}^{\frac{1}{k}}(t, x) q_{x t}(t, x) \\
= & q_{x}^{\frac{k+1}{k}}(t, x)\left[y_{t}(t, q(t, x))+(k+1) u^{k-1} u_{x} y(t, q(t, x))+y_{x}(t, q(t, x)) u^{k}\right] \\
= & -\lambda y(t, q(t, x)) q_{x}^{\frac{k+1}{k}}(t, x) \\
= & -\lambda P(t),
\end{aligned}
$$

the solution of ordinary differential equation is $P(t)=P(0) e^{-\lambda t}$. Since $q(0, x)=x, q_{x}(0, x)=$ 1 , we have

$$
y(t, q(t, x)) q_{x}^{\frac{k+1}{k}}(t, x)=y_{0}(x) e^{-\lambda t} .
$$

This concludes the proof.

Lemma 2.4 Let $u(t, x)$ be the solution to (2.2). Then we have

$$
\int_{R}\left(u^{2}+u_{x}^{2}\right) d x=e^{-2 \lambda t} \int_{R}\left(u_{0}^{2}+u_{0 x}^{2}\right) d x
$$

Proof When $\lambda=0$, Lemma 2.4 is a case of Lemma 2.8 in Zhao et al. [21]. The proof carries over with a slight modification and we present it here for the reader's convenience.

Thanks to $y=u-u_{x x}$ and integrating by parts, we have

$$
\int_{R} y u d x=\int_{R}\left(u^{2}+u_{x}^{2}\right) d x
$$

thus,

$$
\begin{aligned}
& \frac{d}{d t} \int_{R}\left(u^{2}+u_{x}^{2}\right) d x+2 \lambda \int_{R}\left(u^{2}+u_{x}^{2}\right) d x \\
& \quad=\frac{d}{d t} \int_{R} y u d x+2 \lambda \int_{R} y u d x \\
& \quad=\int_{R}\left(y u_{t}+u y_{t}\right) d x+2 \lambda \int_{R} y u d x .
\end{aligned}
$$

Together with (2.1) and (2.2), on integration by parts we have

$$
\begin{aligned}
& \int_{R}\left(y u_{t}+u y_{t}\right) d x+2 \lambda \int_{R} y u d x \\
& =-\int_{R}\left[u^{k+1} y_{x}+(k+1) u^{k} u_{x} y\right] d x-\int_{R} u^{k} u_{x} y \\
& \quad-\int_{R} y G *\left[k(k-1) u^{k-2} u_{x}^{3}+(2 k-1) u^{k-1} u_{x} u_{x x}+(k+1) u^{k} u_{x}\right] d x \\
& =0 .
\end{aligned}
$$


Therefore,

$$
\frac{d}{d t} \int_{R}\left(u^{2}+u_{x}^{2}\right) d x+2 \lambda \int_{R}\left(u^{2}+u_{x}^{2}\right) d x=0 .
$$

Integrating with respect to $t$ from 0 to $t$, we get the desired conclusion.

Following the proof of Lemma 2.9 given by Zhao et al. in [21], we can obtain a similar blow-up result of the solution to the Cauchy problem (2.2).

Theorem 2.1 Let $u_{0}(x) \in H^{s}(R), s>\frac{3}{2}$, and $T=T\left(u_{0}\right)>0$ be the maximal existence time of the corresponding solution $u(t, x)$ to $(2.2)$, then $u(t, x)$ blows up if and only if

$$
\limsup _{t \rightarrow T}\left\|u_{x}(t, x)\right\|_{L^{\infty}}=+\infty
$$

\section{Blow-up and global existence}

Following the local existence Theorem 2.1, we will give our main result on the blow-up property of solution to (2.2). We first give a sufficient condition to guarantee that the solution blows up at a finite time.

Theorem 3.1 Let $u_{0}(x) \in H^{s}(R), s>\frac{3}{2}$, and $T=T\left(u_{0}\right)>0$ be the maximal existence time of the corresponding solution $u(t, x)$ to (2.2). Assume $k=1$ or $k=2 n, n$ is a positive integer, if there exists an $x_{0} \in R$ such that $y_{0}(x)=\left(1-\partial_{x}^{2}\right) u_{0}(x)$ satisfies

$$
y_{0}(x) \geq 0 \quad \text { for }\left(-\infty, x_{0}\right) \text { and } y_{0}(x) \leq 0 \quad \text { for }\left(x_{0},+\infty\right)
$$

and

$$
u_{0}^{k-1}\left(x_{0}\right) u_{0 x}\left(x_{0}\right)<-k \lambda-\sqrt{\frac{1}{2^{k}}\left\|u_{0}\right\|_{H^{1}}^{2 k}+k^{2} \lambda^{2}} .
$$

Then the corresponding solution to (2.2) with initial data $u_{0}(x)$ blows up at finite time $T$ with

$$
T \leq \min \left\{\frac{-2}{(1-\delta) m(0)}, \frac{1}{A} \ln \frac{m(0)-A}{m(0)+A}\right\}
$$

where $0<\delta<1$, such that

$$
-\sqrt{\delta} m(0)=\sqrt{\frac{1}{2^{k}}\left\|u_{0}\right\|_{H^{1}}^{2 k}+k^{2} \lambda^{2}}
$$

and

$$
A=\sqrt{\frac{1}{2^{k}}\left\|u_{0}\right\|_{H^{1}}^{2 k}+k^{2} \lambda^{2}}, \quad m(0)=u_{0}^{k-1}\left(x_{0}\right) u_{0 x}\left(x_{0}\right)+k \lambda .
$$

Proof For $k=1$, the result can be found in Wu and Yin [10]. We just show that the results hold for $k=2 n, n \in N$, and the initial data $u_{0} \in H^{3}(R)$, for the general case we can use the smooth approximate technique and denseness. 
Let $T>0$ be the maximal existence time of the solution $u(t, x)$ to $(2.2)$ with initial data $u_{0}(x)$. Thanks to (2.4), (2.5), and (3.1), we have $y\left(t, q\left(t, x_{0}\right)\right)=0$, and, for all $t>0$, we have

$$
\begin{aligned}
& y(t, q(t, x)) \geq 0, \quad \text { for } x \in\left(-\infty, x_{0}\right), \\
& y(t, q(t, x)) \leq 0, \quad \text { for } x \in\left(x_{0},+\infty\right) .
\end{aligned}
$$

With the help of $u(\cdot, x)=G * y(\cdot, x), x \in R$, we have

$$
u(t, x)=\frac{1}{2} e^{-x} \int_{-\infty}^{x} e^{\xi} y(t, \xi) d \xi+\frac{1}{2} e^{x} \int_{x}^{+\infty} e^{-\xi} y(t, \xi) d \xi
$$

and

$$
u_{x}(t, x)=-\frac{1}{2} e^{-x} \int_{-\infty}^{x} e^{\xi} y(t, \xi) d \xi+\frac{1}{2} e^{x} \int_{x}^{+\infty} e^{-\xi} y(t, \xi) d \xi .
$$

After direct calculations we get

$$
\begin{aligned}
& u(t, x)+u_{x}(t, x)=e^{x} \int_{x}^{\infty} e^{-\xi} y(\xi, t) d \xi \\
& u(t, x)-u_{x}(t, x)=e^{-x} \int_{-\infty}^{x} e^{\xi} y(\xi, t) d \xi
\end{aligned}
$$

and

$$
u(t, x) u_{x}(t, x)=-\frac{1}{4} e^{-2 x}\left(\int_{-\infty}^{x} e^{\xi} y(t, \xi) d \xi\right)^{2}+\frac{1}{4} e^{2 x}\left(\int_{x}^{+\infty} e^{-\xi} y(t, \xi) d \xi\right)^{2}
$$

Thanks to Lemma 2.1, $u_{0}(x) \in H^{3}(R)$ implies that

$$
u(t, x) \in C\left([0, T) ; H^{3}(R)\right) \cap C^{1}\left([0, T) ; H^{2}(R)\right),
$$

then $u(t, \cdot) \in C^{2}(R), u_{t}(t, x) \in C\left([0, T) ; H^{2}(R)\right)$, and $u_{x t}(t, x) \in C\left([0, T) ; H^{1}(R)\right)$.

From (3.6) to (3.8) we have

$$
\begin{aligned}
\frac{d}{d t}[ & \left.\left(k u^{k-1} u_{x}\right)\left(t, q\left(t, x_{0}\right)\right)\right] \\
= & k(k-2) u^{k-2} u_{x} u_{t}+k u^{k-2} \frac{d}{d t}\left(u u_{x}\right) \\
= & k(k-2) u^{k-2} u_{x} u_{t}+k u^{k-2} \frac{d}{d t}\left[-\frac{1}{4} e^{-2 q\left(t, x_{0}\right)}\left(\int_{-\infty}^{q\left(t, x_{0}\right)} e^{\xi} y(\xi, t) d \xi\right)^{2}\right. \\
& \left.+\frac{1}{4} e^{2 q\left(t, x_{0}\right)}\left(\int_{q\left(t, x_{0}\right)}^{+\infty} e^{-\xi} y(\xi, t) d \xi\right)^{2}\right] \\
= & k(k-2) u^{k-2} u_{x} u_{t}+k u^{k-2}\left[\frac{1}{2} e^{-2 q\left(t, x_{0}\right)}\left(\int_{-\infty}^{q\left(t, x_{0}\right)} e^{\xi} y(\xi, t) d \xi\right)^{2} q_{t}\left(t, x_{0}\right)\right. \\
& -\frac{1}{2} e^{-q\left(t, x_{0}\right)}\left(\int_{-\infty}^{q\left(t, x_{0}\right)} e^{\xi} y(\xi, t) d \xi\right) y\left(t, q\left(t, x_{0}\right)\right) q_{t}\left(t, x_{0}\right)
\end{aligned}
$$




$$
\begin{aligned}
& -\frac{1}{2} e^{-2 q\left(t, x_{0}\right)}\left(\int_{-\infty}^{q\left(t, x_{0}\right)} e^{\xi} y(\xi, t) d \xi\right)\left(\int_{-\infty}^{q\left(t, x_{0}\right)} e^{\xi} y_{t}(\xi, t) d \xi\right) \\
& +\frac{1}{2} e^{2 q\left(t, x_{0}\right)}\left(\int_{q\left(t, x_{0}\right)}^{+\infty} e^{-\xi} y(\xi, t) d \xi\right)^{2} q_{t}\left(t, x_{0}\right) \\
& +\frac{1}{2} e^{q\left(t, x_{0}\right)}\left(\int_{q\left(t, x_{0}\right)}^{\infty} e^{-\xi} y(\xi, t) d \xi\right) y\left(t, q\left(t, x_{0}\right)\right) q_{t}\left(t, x_{0}\right) \\
& \left.+\frac{1}{2} e^{2 q\left(t, x_{0}\right)}\left(\int_{q\left(t, x_{0}\right)}^{+\infty} e^{-\xi} y(\xi, t) d \xi\right)\left(\int_{q\left(t, x_{0}\right)}^{+\infty} e^{-\xi} y_{t}(\xi, t) d \xi\right)\right] .
\end{aligned}
$$

Notice $y\left(t, q\left(t, x_{0}\right)\right)=0$, using (2.3), (3.6), and (3.7) we have

$$
\begin{aligned}
\frac{d}{d t}[ & \left.\left(k u^{k-1} u_{x}\right)\left(t, q\left(t, x_{0}\right)\right)\right] \\
= & k(k-2) u^{k-2} u_{x} u_{t}+k u^{k-2}\left[\frac{1}{2} e^{-2 q\left(t, x_{0}\right)}\left(\int_{-\infty}^{q\left(t, x_{0}\right)} e^{\xi} y(\xi, t) d \xi\right)^{2} q_{t}\left(t, x_{0}\right)\right. \\
& -\frac{1}{2} e^{-2 q\left(t, x_{0}\right)}\left(\int_{-\infty}^{q\left(t, x_{0}\right)} e^{\xi} y(\xi, t) d \xi\right)\left(\int_{-\infty}^{q\left(t, x_{0}\right)} e^{\xi} y_{t}(\xi, t) d \xi\right) \\
& +\frac{1}{2} e^{2 q\left(t, x_{0}\right)}\left(\int_{q\left(t, x_{0}\right)}^{+\infty} e^{-\xi} y(\xi, t) d \xi\right)^{2} q_{t}\left(t, x_{0}\right) \\
& \left.+\frac{1}{2} e^{2 q\left(t, x_{0}\right)}\left(\int_{q\left(t, x_{0}\right)}^{+\infty} e^{-\xi} y(\xi, t) d \xi\right)\left(\int_{q\left(t, x_{0}\right)}^{+\infty} e^{-\xi} y_{t}(\xi, t) d \xi\right)\right] \\
= & k(k-2) u^{k-2} u_{x} u_{t}+\frac{k}{2} u^{2 k-2}\left(u-u_{x}\right)^{2}+\frac{k}{2} u^{2 k-2}\left(u+u_{x}\right)^{2} \\
& -\frac{k}{2} u^{k-2}\left(u-u_{x}\right) e^{-q\left(t, x_{0}\right)} \int_{-\infty}^{q\left(t, x_{0}\right)} e^{\xi} y_{t}(\xi, t) d \xi \\
& +\frac{k}{2} u^{k-2}\left(u+u_{x}\right) e^{q\left(t, x_{0}\right)} \int_{q\left(t, x_{0}\right)}^{+\infty} e^{-\xi} y_{t}(\xi, t) d \xi
\end{aligned}
$$

Now we calculate the first term on the right hand side of (3.10). From (2.3), (3.4), and $y\left(t, q\left(t, x_{0}\right)\right)=0$, we obtain

$$
\begin{aligned}
k(k-2) & \left(u^{k-2} u_{x} u_{t}\right)\left(t, q\left(t, x_{0}\right)\right) \\
= & k(k-2) u^{k-2} u_{x}\left[-\frac{1}{2} e^{-q\left(t, x_{0}\right)} q_{t}\left(t, x_{0}\right) \int_{-\infty}^{q\left(t, x_{0}\right)} e^{\xi} y(\xi, t) d \xi\right. \\
& +\frac{1}{2} y\left(t, q\left(t, x_{0}\right)\right) q_{t}\left(t, x_{0}\right)+\frac{1}{2} e^{-q\left(t, x_{0}\right)} \int_{-\infty}^{q\left(t, x_{0}\right)} e^{\xi} y_{t}(\xi, t) d \xi \\
& +\frac{1}{2} e^{q\left(t, x_{0}\right)} q_{t}\left(t, x_{0}\right) \int_{q\left(t, x_{0}\right)}^{+\infty} e^{-\xi} y(\xi, t) d \xi-\frac{1}{2} y\left(t, q\left(t, x_{0}\right)\right) q_{t}\left(t, x_{0}\right) \\
& \left.+\frac{1}{2} e^{q\left(t, x_{0}\right)} \int_{q\left(t, x_{0}\right)}^{+\infty} e^{-\xi} y_{t}(\xi, t) d \xi\right] \\
= & k(k-2) u^{2 k-2} u_{x}^{2}+k(k-2) u^{k-2} u_{x}\left[\frac{1}{2} e^{-q\left(t, x_{0}\right)} \int_{-\infty}^{q\left(t, x_{0}\right)} e^{\xi} y_{t}(\xi, t) d \xi\right. \\
& \left.+\frac{1}{2} e^{q\left(t, x_{0}\right)} \int_{q\left(t, x_{0}\right)}^{+\infty} e^{-\xi} y_{t}(\xi, t) d \xi\right] \cdot
\end{aligned}
$$


Substituting (3.11) into (3.10) yields

$$
\begin{aligned}
\frac{d}{d t}\left[\left(k u^{k-1} u_{x}\right)\left(t, q\left(t, x_{0}\right)\right)\right] \\
=k(k-2) u^{2 k-2} u_{x}^{2}+\frac{k}{2} u^{2 k-2}\left(u-u_{x}\right)^{2}+\frac{k}{2} u^{2 k-2}\left(u+u_{x}\right)^{2} \\
\quad-\frac{k}{2} u^{k-2}\left[u-(k-1) u_{x}\right] e^{-q\left(t, x_{0}\right)} \int_{-\infty}^{q\left(t, x_{0}\right)} e^{\xi} y_{t}(\xi, t) d \xi \\
+\frac{k}{2} u^{k-2}\left[u+(k-1) u_{x}\right] e^{q\left(t, x_{0}\right)} \int_{q\left(t, x_{0}\right)}^{+\infty} e^{-\xi} y_{t}(\xi, t) d \xi
\end{aligned}
$$

By (2.1), integration by parts gives

$$
\begin{aligned}
& e^{-q\left(t, x_{0}\right)} \int_{-\infty}^{q\left(t, x_{0}\right)} e^{\xi} y_{t}(\xi, t) d \xi \\
& =-e^{-q\left(t, x_{0}\right)}\left[\int_{-\infty}^{q\left(t, x_{0}\right)} e^{\xi}\left(y u^{k}\right)_{\xi} d \xi+\int_{-\infty}^{q\left(t, x_{0}\right)} e^{\xi} d y u^{k-1} u_{\xi} \xi+\lambda \int_{-\infty}^{q\left(t, x_{0}\right)} e^{\xi} y d \xi\right] \\
& =e^{-q\left(t, x_{0}\right)} \int_{-\infty}^{q\left(t, x_{0}\right)} e^{\xi} y u^{k-1}\left(u-u_{\xi}\right) d \xi-\lambda e^{-q\left(t, x_{0}\right)} \int_{-\infty}^{q\left(t, x_{0}\right)} e^{\xi} y d \xi .
\end{aligned}
$$

Thanks to $y=u-u_{x x}$ and (3.7), we get

$$
\begin{aligned}
& e^{-q\left(t, x_{0}\right)} \int_{-\infty}^{q\left(t, x_{0}\right)} e^{\xi} y_{t}(\xi, t) d \xi \\
& =e^{-q\left(t, x_{0}\right)} \int_{-\infty}^{q\left(t, x_{0}\right)} e^{\xi}\left(u-u_{\xi \xi}\right) u^{k-1}\left(u-u_{\xi}\right) d \xi-\lambda e^{-q\left(t, x_{0}\right)} \int_{-\infty}^{q\left(t, x_{0}\right)} e^{\xi} y d \xi \\
& =e^{-q\left(t, x_{0}\right)} \int_{-\infty}^{q\left(t, x_{0}\right)} e^{\xi} u^{k-1}\left(u^{2}-u u_{\xi \xi}-u u_{\xi}+u_{\xi} u_{\xi \xi}\right) d \xi-\lambda e^{-q\left(t, x_{0}\right)} \int_{-\infty}^{q\left(t, x_{0}\right)} e^{\xi} y d \xi \\
& =e^{-q\left(t, x_{0}\right)} \int_{-\infty}^{q\left(t, x_{0}\right)} e^{\xi}\left(u^{k-1} u_{\xi} u_{\xi \xi}-u^{k} u_{\xi \xi}-u^{k} u_{\xi}+u^{k+1}\right) d \xi-\lambda e^{-q\left(t, x_{0}\right)} \int_{-\infty}^{q\left(t, x_{0}\right)} e^{\xi} y d \xi .
\end{aligned}
$$

Since

$$
\begin{aligned}
& \left(u^{k} u_{x}\right)_{x}=k u^{k-1} u_{x}^{2}+u^{k} u_{x x}, \\
& \left(u^{k-1} u_{x}^{2}\right)_{x}=(k-1) u^{k-2} u_{x}^{3}+2 u^{k-1} u_{x} u_{x x},
\end{aligned}
$$

we have

$$
\begin{aligned}
& e^{-q\left(t, x_{0}\right)} \int_{-\infty}^{q\left(t, x_{0}\right)} e^{\xi} u^{k-1} u_{\xi} u_{\xi \xi} d \xi \\
& =\frac{1}{2} e^{-q\left(t, x_{0}\right)} \int_{-\infty}^{q\left(t, x_{0}\right)} e^{\xi}\left[\left(u^{k-1} u_{\xi}^{2}\right)_{\xi}-(k-1) u^{k-2} u_{\xi}^{3}\right] d \xi \\
& =\frac{1}{2}\left(u^{k-1} u_{x}^{2}\right)\left(t, q\left(t, x_{0}\right)\right)-\frac{1}{2} e^{-q\left(t, x_{0}\right)} \int_{-\infty}^{q\left(t, x_{0}\right)} e^{\xi}\left[u^{k-1} u_{\xi}^{2}+(k-1) u^{k-2} u_{\xi}^{3}\right] d \xi
\end{aligned}
$$


and

$$
\begin{aligned}
& -e^{-q\left(t, x_{0}\right)} \int_{-\infty}^{q\left(t, x_{0}\right)} e^{\xi} u^{k} u_{\xi \xi} d \xi \\
& =-e^{-q\left(t, x_{0}\right)} \int_{-\infty}^{q\left(t, x_{0}\right)} e^{\xi}\left[\left(u^{k} u_{\xi}\right)_{\xi}-k u^{k-1} u_{\xi}^{2}\right] d \xi \\
& =-\left(u^{k} u_{x}\right)\left(t, q\left(t, x_{0}\right)\right)+e^{-q\left(t, x_{0}\right)} \int_{-\infty}^{q\left(t, x_{0}\right)} e^{\xi}\left(u^{k} u_{\xi}+k u^{k-1} u_{\xi}^{2}\right) d \xi
\end{aligned}
$$

therefore

$$
\begin{aligned}
e^{-q\left(t, x_{0}\right)} & \int_{-\infty}^{q\left(t, x_{0}\right)} e^{\xi} y_{t}(\xi, t) d \xi \\
= & -\left(u^{k} u_{x}\right)\left(t, q\left(t, x_{0}\right)\right)+\frac{1}{2}\left(u^{k-1} u_{x}^{2}\right)\left(t, q\left(t, x_{0}\right)\right) \\
& +e^{-q\left(t, x_{0}\right)} \int_{-\infty}^{q\left(t, x_{0}\right)} e^{\xi}\left(u^{k+1}+\frac{2 k-1}{2} u^{k-1} u_{\xi}^{2}-\frac{k-1}{2} u^{k-2} u_{\xi}^{3}\right) d \xi \\
& -\lambda\left(u-u_{x}\right)\left(t, q\left(t, x_{0}\right)\right) \\
= & -\left(u^{k} u_{x}\right)\left(t, q\left(t, x_{0}\right)\right)+\frac{1}{2}\left(u^{k-1} u_{x}^{2}\right)\left(t, q\left(t, x_{0}\right)\right)-\lambda\left(u-u_{x}\right)\left(t, q\left(t, x_{0}\right)\right) \\
& +e^{-q\left(t, x_{0}\right)} \int_{-\infty}^{q\left(t, x_{0}\right)} \frac{e^{\xi}}{2}\left[u^{k+1}+u^{k-2}\left(u-u_{\xi}\right)^{2}\left(u-(k-1) u_{\xi}\right)+(k+1) u^{k} u_{\xi}\right] d \xi \\
= & -\left(u^{k} u_{x}\right)\left(t, q\left(t, x_{0}\right)\right)+\frac{1}{2}\left(u^{k-1} u_{x}^{2}\right)\left(t, q\left(t, x_{0}\right)\right)-\lambda\left(u-u_{x}\right)\left(t, q\left(t, x_{0}\right)\right) \\
& +\frac{1}{2} u^{k+1}\left(t, q\left(t, x_{0}\right)\right)+e^{-q\left(t, x_{0}\right)} \int_{-\infty}^{q\left(t, x_{0}\right)} \frac{e^{\xi}}{2} u^{k-2}\left(u-u_{\xi}\right)^{2}\left[u-(k-1) u_{\xi}\right] d \xi .
\end{aligned}
$$

Thanks to (3.3),

$$
\begin{aligned}
& y(t, q(t, x)) \geq 0, \quad \text { for } x \in\left(-\infty, x_{0}\right), t \geq 0, \\
& y(t, q(t, x)) \leq 0, \quad \text { for } x \in\left(x_{0},+\infty\right), t \geq 0,
\end{aligned}
$$

together with (3.6) and (3.7),

$$
\begin{aligned}
& u\left(t, q\left(t, x_{0}\right)\right)+u_{x}\left(t, q\left(t, x_{0}\right)\right)=e^{q\left(t, x_{0}\right)} \int_{q\left(t, x_{0}\right)}^{\infty} e^{-\xi} y(\xi, t) d \xi \leq 0, \\
& u\left(t, q\left(t, x_{0}\right)\right)-u_{x}\left(t, q\left(t, x_{0}\right)\right)=e^{-q\left(t, x_{0}\right)} \int_{-\infty}^{q\left(t, x_{0}\right)} e^{\xi} y(\xi, t) d \xi \geq 0,
\end{aligned}
$$

we have $u_{x}\left(t, q\left(t, x_{0}\right)\right) \leq 0$.

Noticing $k=2 n, n$ is a positive integer, we have

$$
u-(k-1) u_{x} \geq u-u_{x} \geq 0, \quad \forall x \in\left(-\infty, q\left(t, x_{0}\right)\right),
$$

hence

$$
\int_{-\infty}^{q\left(x_{0}, t\right)} \frac{e^{\xi}}{2} u^{k-2}\left(u-u_{\xi}\right)^{2}\left[u-(k-1) u_{\xi}\right] d \xi \geq 0
$$


This implies

$$
\begin{aligned}
e^{-q\left(t, x_{0}\right)} \int_{-\infty}^{q\left(t, x_{0}\right)} e^{\xi} y_{t}(\xi, t) d \xi \geq & -\left(u^{k} u_{x}\right)\left(t, q\left(t, x_{0}\right)\right)+\frac{1}{2}\left(u^{k-1} u_{x}^{2}\right)\left(t, q\left(t, x_{0}\right)\right) \\
& +\frac{1}{2} u^{k+1}\left(t, q\left(t, x_{0}\right)\right)-\lambda\left(u-u_{x}\right)\left(t, q\left(t, x_{0}\right)\right) .
\end{aligned}
$$

Similarly, we repeat the above calculations and obtain

$$
\begin{aligned}
e^{q\left(t, x_{0}\right)} & \int_{q\left(t, x_{0}\right)}^{+\infty} e^{-\xi} y_{t}(\xi, t) d \xi \\
= & -\left(u^{k} u_{x}\right)\left(t, q\left(t, x_{0}\right)\right)-\frac{1}{2}\left(u^{k-1} u_{x}^{2}\right)\left(t, q\left(t, x_{0}\right)\right) \\
& -\lambda\left(u+u_{x}\right)\left(t, q\left(t, x_{0}\right)\right)-\frac{1}{2} u^{k+1}\left(t, q\left(t, x_{0}\right)\right) \\
& -e^{-q\left(t, x_{0}\right)} \int_{q\left(t, x_{0}\right)}^{+\infty} \frac{e^{\xi}}{2} u^{k-2}\left(u+u_{\xi}\right)^{2}\left[u+(k-1) u_{\xi}\right] d \xi
\end{aligned}
$$

Since $u_{x}\left(t, q\left(t, x_{0}\right)\right) \leq 0$, then

$$
u+(k-1) u_{x} \leq u+u_{x} \leq 0, \quad \forall x \in\left(q\left(t, x_{0}\right),+\infty\right),
$$

we have

$$
\begin{aligned}
e^{q\left(t, x_{0}\right)} \int_{q\left(t, x_{0}\right)}^{+\infty} e^{-\xi} y_{t}(\xi, t) d \xi \geq & -\left(u^{k} u_{x}\right)\left(t, q\left(t, x_{0}\right)\right)-\frac{1}{2}\left(u^{k-1} u_{x}^{2}\right)\left(t, q\left(t, x_{0}\right)\right) \\
& -\frac{1}{2} u^{k+1}\left(t, q\left(t, x_{0}\right)\right)-\lambda\left(u+u_{x}\right)\left(t, q\left(t, x_{0}\right)\right) .
\end{aligned}
$$

Inserting (3.15) and (3.17) into (3.12), we get

$$
\begin{aligned}
\frac{d}{d t}[ & \left.\left(u^{k-1} u_{x}\right)\left(t, q\left(t, x_{0}\right)\right)\right] \\
& \leq(k-2) u^{2 k-2} u_{x}^{2}+\frac{1}{2} u^{2 k-2}\left(u-u_{x}\right)^{2}+\frac{1}{2} u^{2 k-2}\left(u+u_{x}\right)^{2} \\
& \quad-\frac{1}{2} u^{k-2}\left[u-(k-1) u_{x}\right]\left[-u^{k} u_{x}+\frac{1}{2} u^{k-1} u_{x}^{2}+\frac{1}{2} u^{k+1}-\lambda\left(u-u_{x}\right)\right] \\
& +\frac{1}{2} u^{k-2}\left[u+(k-1) u_{x}\right]\left[-u^{k} u_{x}-\frac{1}{2} u^{k-1} u_{x}^{2}-\frac{1}{2} u^{k+1}-\lambda\left(u+u_{x}\right)\right] \\
= & \frac{1}{2} u^{2 k}\left(t, q\left(t, x_{0}\right)\right)-\frac{1}{2}\left(u^{2 k-2} u_{x}^{2}\right)\left(t, q\left(t, x_{0}\right)\right)-\lambda k\left(u^{k-1} u_{x}\right)\left(t, q\left(t, x_{0}\right)\right) .
\end{aligned}
$$

Thanks to the Cauchy-Schwartz inequality,

$$
\begin{aligned}
2 u^{2}(x, t) & =2\left(\int_{-\infty}^{x} u u_{x} d x-\int_{x}^{+\infty} u u_{x} d x\right) \\
& \leq \int_{-\infty}^{x}\left(u^{2}+u_{x}^{2}\right) d x+\int_{x}^{+\infty}\left(u^{2}+u_{x}^{2}\right) d x \\
& =\|u(x, t)\|_{H^{1}}^{2},
\end{aligned}
$$


from Lemma 2.4, we have

$$
2 u^{2}(t, x) \leq\|u(t, x)\|_{H^{1}}^{2}=e^{-2 \lambda t}\left\|u_{0}\right\|_{H^{1}}^{2} \leq\left\|u_{0}\right\|_{H^{1}}^{2}
$$

then

$$
\|u(t, x)\|_{L^{\infty}} \leq \frac{\sqrt{2}}{2}\left\|u_{0}\right\|_{H^{1}} .
$$

Combining (3.18) with (3.20), we have

$$
\begin{aligned}
& \frac{d}{d t}\left[\left(u^{k-1} u_{x}\right)\left(t, q\left(t, x_{0}\right)\right)\right] \\
& \quad \leq \frac{1}{2^{k+1}}\left\|u_{0}\right\|_{H^{1}}^{2 k}-\frac{1}{2}\left(u^{2 k-2} u_{x}^{2}\right)\left(t, q\left(t, x_{0}\right)\right)-\lambda k\left(u^{k-1} u_{x}\right)\left(t, q\left(t, x_{0}\right)\right) .
\end{aligned}
$$

We now define a function

$$
m(t)=\left(u^{k-1} u_{x}\right)\left(t, q\left(t, x_{0}\right)\right)+\lambda k
$$

since $\left(u^{k-1} u_{x}\right)\left(t, q\left(t, x_{0}\right)\right)$ is continuously differentiable on $[0, T), m(t)$ is continuously differentiable on $[0, T)$, from (3.21), we obtain

$$
\begin{aligned}
\frac{d m(t)}{d t} & \leq \frac{1}{2^{k+1}}\left\|u_{0}\right\|_{H^{1}}^{2 k}-\frac{1}{2}(m(t)-\lambda k)^{2}-\lambda k(m(t)-\lambda k) \\
& =-\frac{1}{2} m^{2}(t)+\frac{1}{2^{k+1}}\left\|u_{0}\right\|_{H^{1}}^{2 k}+\frac{\lambda^{2} k^{2}}{2} .
\end{aligned}
$$

By the assumption

$$
m(0)=u_{0}^{k-1}\left(x_{0}\right) u_{0 x}\left(x_{0}\right)+k \lambda<-\sqrt{\frac{1}{2^{k}}\left\|u_{0}\right\|_{H^{1}}^{2 k}+k^{2} \lambda^{2}}
$$

we have $m^{2}(0)>\frac{1}{2^{k}}\left\|u_{0}\right\|_{H^{1}}^{2 k}+k^{2} \lambda^{2}$.

We claim that

$$
m(t)<-\sqrt{\frac{1}{2^{k}}\left\|u_{0}\right\|_{H^{1}}^{2 k}+k^{2} \lambda^{2}}, \quad \forall t \in[0, T) .
$$

Otherwise, if (3.23) is not true, by the continuity of $m(t)$, there exists a $t_{0} \in(0, T)$ such that, for all $t \in\left[0, t_{0}\right)$,

$$
m^{2}(t)>\frac{1}{2^{k}}\left\|u_{0}\right\|_{H^{1}}^{2 k}+k^{2} \lambda^{2}
$$

and

$$
m^{2}\left(t_{0}\right)=\frac{1}{2^{k}}\left\|u_{0}\right\|_{H^{1}}^{2 k}+k^{2} \lambda^{2}
$$

Combining (3.22) and (3.24), we have, for all $t \in\left[0, t_{0}\right]$,

$$
\frac{d m(t)}{d t} \leq 0
$$


since $m(t)$ is continuously differentiable on $\left[0, t_{0}\right]$, integrating (3.26) with respect to $t$ from 0 to $t_{0}$, we have

$$
m\left(t_{0}\right) \leq m(0)=u_{0}^{k-1}\left(x_{0}\right) u_{0 x}\left(x_{0}\right)+k \lambda<-\sqrt{\frac{1}{2^{k}}\left\|u_{0}\right\|_{H^{1}}^{2 k}+k^{2} \lambda^{2}} .
$$

Recalling (3.24), we get the desired contradiction, which concludes the proof of the claim.

Since $m(t)$ is continuously differentiable and strictly decreasing on $[0, T)$, we can choose $\delta \in(0,1)$ such that

$$
-\sqrt{\delta} m(0)=\sqrt{\frac{1}{2^{k}}\left\|u_{0}\right\|_{H^{1}}^{2 k}+k^{2} \lambda^{2}}
$$

thanks to (3.22) and (3.28), we have, for all $t \in[0, T)$,

$$
\frac{d m(t)}{d t} \leq-\frac{1-\delta}{2} m^{2}(t)
$$

Since $m(t)$ is continuously differentiable and strictly negative on $[0, T)$, hence $\frac{1}{m(t)}$ is continuously differentiable on $[0, T)$, and

$$
\frac{d}{d t}\left(\frac{1}{m(t)}\right)=-\frac{1}{m^{2}(t)} \frac{d m(t)}{d t}>\frac{1-\delta}{2}, \quad \forall t \in[0, T)
$$

Integrating with respect to $t$ over $[0, T]$ on both sides of (3.30) yields

$$
\frac{1}{m(t)}-\frac{1}{m(0)}>\frac{1-\delta}{2} t, \quad \forall t \in[0, T) .
$$

Since $m(t)<0$ on $[0, T)$, we know that the maximal existence time is

$$
T^{*}=-\frac{2}{(1-\delta) m(0)}<+\infty
$$

such that

$$
\lim _{t \uparrow T^{*}} m(t) \leq \lim _{t \uparrow T^{*}} \frac{2}{(1-\delta)\left(t-T^{*}\right)}=-\infty
$$

Since

$$
\inf _{x \in R}\left(u^{k-1} u_{x}\right)(t, x) \leq\left(u^{k-1} u_{x}\right)\left(t, q\left(t, x_{0}\right)\right)=m(t)-\lambda k
$$

this implies

$$
\lim _{t \uparrow T^{*}} \inf _{x \in R}\left(u^{k-1} u_{x}\right)(x, t) \leq \lim _{t \uparrow T^{*}}\left[\frac{2}{(1-\delta)\left(t-T^{*}\right)}-\lambda k\right]=-\infty
$$

For $A=\sqrt{\frac{1}{2^{k}}\left\|u_{0}\right\|_{H^{1}}^{2 k}+k^{2} \lambda^{2}}>0$, from (3.22), we have

$$
\frac{1}{2 A}\left(\frac{1}{m(t)-A}-\frac{1}{m(t)+A}\right) d m(t)=\frac{d m(t)}{m^{2}(t)-A^{2}} \leq-\frac{1}{2} d t
$$


that is,

$$
\left(\frac{1}{m(t)-A}-\frac{1}{m(t)+A}\right) d m(t) \leq-A d t
$$

integrating with respect to $t$ over $[0, t]$ yields

$$
\begin{aligned}
& \ln \frac{m(t)-A}{m(t)+A}-\ln \frac{m(0)-A}{m(0)+A} \leq-A t . \\
& \text { As } m(t)<-A<0, \ln \frac{m(t)-A}{m(t)+A}>0, \text { we have } \\
& \quad t \leq \frac{1}{A} \ln \frac{m(0)-A}{m(0)+A}
\end{aligned}
$$

due to $m(0)<-A, \ln \frac{m(0)-A}{m(0)+A}>0$, from (3.32) and (3.35), we can choose

$$
T \leq \min \left\{-\frac{2}{(1-\delta) m(0)}, \frac{1}{A} \ln \frac{m(0)-A}{m(0)+A}\right\}
$$

This completes the proof.

Remark 3.1 The result in Theorem 3.1 contains the cases for $k=1$ : the weakly dissipative Camassa-Holm equation and $k=2$ : the weakly dissipative Novikov equation. We used the method developed by Liu and Yin [22] to deal with the Degasperis-Procesi equation (1.5): $u_{t}-u_{t x x}+4 u u_{x}=3 u_{x} u_{x x}+u u_{x x x}$, but (1.4) contains higher power nonlinear dispersion terms $(k+1) u^{k-1} u_{x} u_{x x}, u^{k} u_{x x x}$, and the nonlinear convection term $(k+2) u^{k} u_{x}$. When the local solution $u(t, x)$ of (2.2) exists, in the proof of its blow-up property we need the sign of $u^{k-2}(t, x)$; see the last term in (3.13). In general, $u(t, x)$ changes the sign for $x \in R$ so we give the condition on the power of nonlinear term $k=2 n, n \in N$ in (1.4). For $k=1$, the last term in (3.13) disappears; for $k=2$, the last term in (3.13) does not contain $u^{k-2}(t, x)$. Therefore, we generalized the blow-up property of the solutions to the Cauchy problem (1.4).

Finally we give a global existence result, thanks to Theorem 2.1, this will be done if we can estimate $\left\|u_{x}(x, t)\right\|_{L^{\infty}}$ is finite.

Theorem 3.2 Let $u_{0}(x) \in H^{s}(R), s>\frac{3}{2}$. If $y_{0}(x)=\left(1-\partial_{x}^{2}\right) u_{0}(x)$ does not change sign on $R$, then the problem (2.2) has a strong solution

$$
u(x, t) \in C\left([0,+\infty) ; H^{s}(R)\right) \cap C^{1}\left([0,+\infty) ; H^{s-1}(R)\right) .
$$

Proof We just consider $s=3$, otherwise we can use the smooth approximate technique and denseness. When $y_{0}(x)=\left(1-\partial_{x}^{2}\right) u_{0}(x) \geq 0$, then from Lemma 2.2 and Lemma 2.3, we can derive that $y(t, x) \geq 0$, for all $[0, T)$.

Due to the positivity of the Green's function $G(x)$ and $u(t, x)=G(x) * y(t, x)$, we obtain $u(t, x) \geq 0$, for all $t \geq 0, u(t, x)+u_{x}(t, x) \geq 0$, and $u(t, x)-u_{x}(t, x) \geq 0$, and these imply that, 
for all $(t, x) \in[0, T) \times R$,

$$
\begin{aligned}
\left|u_{x}(t, x)\right| & \leq u(t, x) \leq\|u(t, x)\|_{L^{\infty}} \\
& \leq \frac{\sqrt{2}}{2}\|u(t, x)\|_{H^{1}}=\frac{\sqrt{2}}{2} e^{-\lambda t}\left\|u_{0}(x)\right\|_{H^{1}} \\
& \leq\left\|u_{0}(x)\right\|_{H^{1}},
\end{aligned}
$$

we obtain $u(t, x) \in C\left([0,+\infty) ; H^{s}(R)\right) \cap C^{1}\left([0,+\infty) ; H^{s-1}(R)\right)$ by Theorem 2.1 .

When $y_{0}(x)=\left(1-\partial_{x}^{2}\right) u_{0}(x) \leq 0$, thanks to Lemma 2.2 and Lemma 2.3, we obtain $y(t, x) \leq$ 0 , for all $[0, T)$. Since $u(t, x)=G(x) * y(t, x)$ and due to the positivity of $G(x)$, we obtain $u(t, x) \leq 0$, for all $t \geq 0, u(t, x)+u_{x}(t, x) \leq 0$, and $u(t, x)-u_{x}(t, x) \leq 0$, and these imply that, for all $(t, x) \in[0, T) \times R$,

$$
\begin{aligned}
\left\|u_{x}(t, x)\right\|_{L^{\infty}} & \leq-u(t, x) \leq\|u(t, x)\|_{L^{\infty}} \\
& \leq \frac{\sqrt{2}}{2}\|u(t, x)\|_{H^{1}}=\frac{\sqrt{2}}{2} e^{-\lambda t}\left\|u_{0}(x)\right\|_{H^{1}} \\
& \leq\left\|u_{0}(x)\right\|_{H^{1}},
\end{aligned}
$$

we obtain $u(t, x) \in C\left([0,+\infty) ; H^{s}(R)\right) \cap C^{1}\left([0,+\infty) ; H^{s-1}(R)\right)$ by Theorem 2.1.

Therefore, we find that the solution exists globally in time.

Competing interests

The authors declare that they have no competing interests.

\section{Authors' contributions}

YG carried out the blow-up property of solutions. YT carried out the global existence of solutions. All authors read and approved the final manuscript.

\section{Author details}

${ }^{1}$ School of Mathematics and Statistics, Huazhong University of Science and Technology, Wuhan, Hubei 430074, P.R. China. ${ }^{2}$ School of Mathematics and Statistics, Xuchang University, Xuchang, Henan 461000, P.R. China.

\section{Acknowledgements}

This work was supported by National Natural Science Foundation of China (grant Nos. 11471129, 11272277).

\section{Received: 17 September 2014 Accepted: 8 December 2014 Published: 18 Dec 2014}

\section{References}

1. Guo, YT, Wang, M, Tang, YB: Higher regularity of global attractor for a damped Benjamin-Bona-Mahony equation on R. Appl. Anal. (2014). doi:10.1080/00036811.2014.946561

2. Camassa, R, Holm, D: An integrable shallow water equation with peaked solitons. Phys. Rev. Lett. 71, 1661-1664 (1993)

3. Constantin, A, Escher, J: Global weak solutions for a shallow water equation. Indiana Univ. Math. J. 47, 1527-1545 (1998)

4. Constantin, A, Escher, J: Wave breaking for nonlocal shallow water equations. Acta Math. 181, 229-243 (1998)

5. Constantin, A, Escher, J: Global existence and blow-up for a shallow water equation. Ann. Sc. Norm. Super. Pisa, Cl. Sci. XXVI(4), 303-328 (1998)

6. Constantin, A: Existence of permanent and breaking waves for a shallow water equation: a geometric approach. Ann. Inst. Fourier (Grenoble) 50, 321-362 (2000)

7. Lai, S, Wu, Y: The local well-posedness and existence of weak solutions for a generalized Camassa-Holm equation. J. Differ. Equ. 248, 2038-2063 (2010)

8. Constantin, A, Escher, J: On the blow-up rate and the blow-up set of breaking waves for a shallow water equation Math. Z. 233, 75-91 (2000)

9. Wu, S, Yin, Z: Blowup, blowup rate and decay of the solution of the weakly dissipative Camassa-Holm equation. J. Math. Phys. 47, 1-12 (2006)

10. Wu, S, Yin, Z: Global existence and blow-up phenomena for the weakly dissipative Camassa-Holm equation. J. Differ. Equ. 246, 4309-4321 (2009) 
11. Lai, S, Wu, Y: Global solutions and blow-up phenomena to a shallow water equations. J. Differ. Equ. 249, 693-706 (2010)

12. Zhou, Y: Blowup of solutions to the DGH equation. J. Funct. Anal. 250, 227-248 (2007)

13. Xin, Z, Zhang, P: On the uniqueness and large time behavior of the weak solution to a shallow water equation. Commun. Partial Differ. Equ. 27(9-10), 1815-1844 (2002)

14. Xin, Z, Zhang, P: On the weak solution to a shallow water equation. Commun. Pure Appl. Math. 53, 1411-1433 (2000)

15. Novikov, V: Generalizations of the Camassa-Holm equation. J. Phys. A 42, 342002 (2009)

16. Lai, S, Li, N, Wu, Y: The existence of global strong and weak solutions for Novikov equation. J. Math. Anal. Appl. 399 , 682-691 (2013)

17. Ni, L, Zhou, Y: Well-posedness and persistence properties for the Novikov equation. J. Differ. Equ. 250, 3002-3201 (2011)

18. Zhao, Y, Li, Y, Yan, W: Local well-posedness and persistence property for the generalized Novikov equation. Discrete Contin. Dyn. Syst., Ser. A 34, 803-820 (2014)

19. Jiang, Z, Ni, L: Blow-up phenomena for the integrable Novikov equation. J. Math. Anal. Appl. 385, $551-558$ (2012)

20. Yan, W, Li, Y, Zhang, Y: Global existence and blow-up phenomena for the weakly dissipative Novikov equation. Nonlinear Anal. 75, 2464-2473 (2012)

21. Zhao, Y, Li, Y, Yan, W: The global weak solutions to the Cauchy problem of the generalized Novikov equation. Appl. Anal. (2014). doi:10.1080/00036811.2014.930826

22. Liu, Y, Yin, Z: On the blow-up phenomena for the Degasperis-Procesi equation. Int. Math. Res. Not. (2007). doi:10.1093/imrn/rnm117

10.1186/1029-242X-2014-514

Cite this article as: Guo and Tang: Blow-up for the weakly dissipative generalized Camassa-Holm equation. Journal of Inequalities and Applications 2014, 2014:514

\section{Submit your manuscript to a SpringerOpen ${ }^{\circ}$ journal and benefit from:}

- Convenient online submission

- Rigorous peer review

- Immediate publication on acceptance

- Open access: articles freely available online

- High visibility within the field

- Retaining the copyright to your article 